\title{
Patient-specific three-dimensional printing in cardiovascular disease
}

\author{
Zhonghua Sun
}

Discipline of Medical Radiation Sciences, School of Molecular and Life Sciences, Curtin University, Perth,

Western Australia, 6845, Australia

\section{Mini Review}

Please cite this paper as: Sun Z. Patient-specific threedimension printing in cardiovascular disease. AMJ 2020;13(4):136-141.

https://doi.org/10.35841/1836-1935.13.4.136-141

\section{Corresponding Author:}

Prof Zhonghua Sun

Discipline of Medical Radiation Sciences, School of Molecular and Life Sciences, Curtin University, Perth, WA, 6845, Australia

Email: z.sun@curtin.edu.au

\section{ABSTRACT}

Three-dimensional (3D) printing has become a widely used technique showing great promise in medical applications. In recent years, patient-specific 3D printed models are shown to play an important role in the domain of cardiovascular disease, ranging from medical education to presurgical planning and simulation of complex cardiovascular procedures, as well as development of optimal computed tomography (CT) imaging protocols. This review article provides a summary of the current applications of using 3D printed cardiovascular models with future research directions highlighted.

\section{Key Words}

Cardiovascular disease, coronary artery, heart, model, protocol, three-dimensional printing, visualisation

\section{Implications for Practice:}

\section{What is known about this subject?}

3D printing is increasingly used in medicine with promising reports in cardiovascular disease.

\section{What new information is offered in this review?}

This review provides a summary of the current status of using
3D printed models in the domain of cardiovascular disease covering aspects from congenital heart disease to structural heart disease, aortic and coronary artery disease. Further, use of 3D printed models to optimise cardiac CT scanning protocols is highlighted to indicate the new and emerging research direction along this path.

3. What are the implications for research, policy, or practice?

3D printed models can be used in many different areas serving as a useful tool for clinicians and medical students and healthcare professionals. Incorporation of 3D printed realistic models into clinical practice will play an important role in clinical decision making and improving patient outcomes.

\section{Introduction}

Three-dimensional (3D) printing has demonstrated great value in medical applications with increasing reports in the literature. The clinical value of using patient-specific or personalised 3D printed models in medicine has expanded well beyond its original dominance in orthopaedic and maxillofacial areas to many others areas, such as cardiovascular and cerebrovascular diseases, different types of tumours (brain, lung, liver and kidney tumours). ${ }^{1-10}$ Of these applications, 3D printed physical models have been reported to be useful in cardiovascular disease, in particular, in congenital heart disease (CHD) due to its complexity and variations among different types of CHD. ${ }^{11-15}$ Further, 3D printed models also compliment conventional visualisation tools in other areas including aortic aneurysm and dissection, coronary artery disease (coronary stenosis and valvular disease), cerebral artery disease and pulmonary artery disease. ${ }^{16-20}$ In the following sections, a detailed review of the literature is provided with a focus on the usefulness of 3D printed models in these applications.

\section{D printing in congenital heart disease}

Use of 3D printing technique in CHD represents a fast evolving area with scientific evidence supporting its both clinical and education value (Figure 1). ${ }^{11}$ Different from 
other applications which are mainly dominated by case reports or case series, there are currently five studies of randomised controlled trials (RCTs) reporting the usefulness of 3D printed models in CHD. ${ }^{11-15}$ of these five RCTs, four of them focused on whether use of 3D printed heart models improved medical student education when compared to the current teaching methods, ${ }^{11-14}$ while the remaining study looked at the comparison of 3D printed model group with conventional group in paediatric residents. ${ }^{15}$ Out of the four RCTs on medical education, three of them showed that 3D printed heart models improved or enhanced medical education in comparison with the current methods, ${ }^{11-13}$ while in the study by Wang et al, ${ }^{14}$ results showed no significant differences in either student's satisfaction or understanding of cardiac structures $(p=0.24-0.48)$. Although 70 per cent of the students from both groups preferred 3D printing as a useful tool for medical education, their study did not conclude the superiority of using 3D printed models over traditional methods in teaching cardiac diseases.

The RCTs on education of paediatric residents presented interesting findings. ${ }^{15}$ 3D printed heart models did not demonstrate advantages over traditional methods in learning and interpreting simple CHD such as ventricular septal defect (VSD), but were found to be advantageous in delineating the complex CHD such as Tetralogy of Fallot (ToF). When visualising and assessing the complexity lesion, 3D printed models add benefits to conventional methods by revealing 3D relationships of complex and multiple components associated with complex CHD. ${ }^{15}$ Despite these promising results, further research is required to determine whether $3 D$ printing is used as a supportive tool or alternative to conventional teaching methods. $^{16}$

There are some single centre and multi-centre studies reporting the clinical value of $3 \mathrm{D}$ printing in $\mathrm{CHD}$, and this has been well discussed in the some general literature reviews and systematic reviews. ${ }^{4,6,17-19}$ Readers are referred to these excellent review articles to get a better understanding of how these 3D printed heart models assist clinical decision making in managing CHD patients. It is worth noting that more studies based on multi-centre experiences with inclusion of a larger cohort are needed to validate the clinical value of 3D printing in $\mathrm{CHD}$, in particular how this exciting technique provides guidance for patient's treatment and improves clinical outcomes as well as cost benefits.

\section{D printing in structural non-congenital heart disease}

3D printing has been shown to be useful in structural noncongenital heart disease, in particular, in the planning of interventional procedures of valvular disease and left atrial appendage (LAA). ${ }^{20-28}$ In the aortic valvular disease, 3D printing is increasingly reported in assisting transcatheter aortic valve replacement (TAVR) planning. ${ }^{21-24}$ In addition to high accuracy of replicating anatomical structures of aortic valve compared to 2D images, 3D printed models can predict procedure-related complications such as coronary occlusion, paravalvular leaks and paravalvular aortic regurgitation following the interventions.

Personalised 3D printed models are also shown valuable in the presurgical planning of LAA occlusion. The 3D printed models are used in different aspects such as device sizing, precise catheter position, pre-procedural simulation and guidance for intra-procedural treatment, thus improving patient outcomes. ${ }^{25-28}$ It should be mentioned that current applications of 3D printed models in TAVR and LAA are limited by case reports or case series, therefore, more studies based on large sample size and multi-centre experience are needed.

\section{D printing in coronary artery and other cardiovascular diseases}

Personalised 3D printed models show potential applications on other cardiovascular diseases including coronary artery disease, cerebrovascular disease, aortic disease such as aortic aneurysm and dissection. ${ }^{5,29-40}$ In coronary artery disease or anomalies, 3D printed models are primarily shown to guide interventional procedures for treating coronary fistula. ${ }^{29-34}$ This is evidenced by case reports in the current literature. 3D printing has great value in preoperative planning and simulation of cerebrovascular disease, mainly cerebral artery aneurysms and a recent systematic review has summarised its applications in this area. ${ }^{5}$

Regarding the usefulness of 3D printed in aortic aneurysm and aortic dissection, its main value lies in preoperative planning and simulation of endovascular stent grafting which is a commonly used less-invasive technique for treatment of aortic aneurysm and aortic dissection. ${ }^{35-40}$ 3D printed aorta models allow the surgeons to practice endovascular procedures which are considered especially useful in dealing with complex aortic lesions, such as aneurysms located in the aortic arch. Its clinical value in training inexperienced surgeons and having the potential to improve outcomes of experienced physicians are also reported in the literature. ${ }^{38-40}$ Creation of $3 D$ printed model of aortic dissection is more challenging than other aortic disease due to thin fibrous membrane of the intimal flap which separates true lumen from false lumen. 
The intimal flap could be easily removed during image segmentation, thus post-processing methods need to be applied to preserve the intimal flap in the 3D printed model (Figure 2).

\section{D printing in optimising CT scanning protocols}

Use of 3D printed models to develop optimal computed tomography protocols (CT) is an emerging research area showing great potential to minimise radiation dose associated with CT scans in imaging cardiovascular disease. This has been explored in utilising 3D printed models for investigation of coronary CT angiography protocols for visualisation and assessment of coronary artery disease including calcified plaques (Figures 3 and 4), ${ }^{41-43}$ coronary stenting in terms of stented lumen and stent wire structures, ${ }^{44,45}$ and pulmonary embolism for detection of thrombus in both main and side pulmonary artery branches. ${ }^{46,47}$ Recent review articles summarise the use of 3D printed models for studying optimal CT protocols and readers are referred to them for detailed information. ${ }^{48,49}$

\section{Summary and conclusion}

Patient-specific or personalised 3D printed models are increasingly used in the domain of cardiovascular disease with promising results reported in the literature. These realistic 3D printed models can be used in various areas ranging from the most common applications of assisting pre-surgical planning and simulation of complex cardiovascular procedures to education of medical students, and emerging areas of optimising CT imaging protocols in the diagnostic assessment of cardiovascular disease. However, some challenges and limitations still exist before the $3 D$ printing technique is incorporated into routine clinical practice. These include commonly recognised issues such as high costs associated with 3D printers and printing materials, time-consuming on image post-processing and segmentation of cardiac images, and limited sample size in the current reports. Large scale studies, preferably multi-centre and randomised controlled trials are desirable to provide robust results so that $3 D$ printing can become an essential component of the daily diagnostic approach.

\section{References}

1. Ryan J, Plasencia J, Richardson R, et al. 3D printing for congenital heart disease: a single site's initial three-year experience. 3D Print Med. 2018;4:10.

2. Giannopoulos AA, Steigner ML, George $E$, et al. Cardiothoracic applications of 3-dimensional printing. J Thorac Imaging. 2016;31:253-272.
3. Witowski J, Wake N, Grochowska A, et al. Investigating accuracy of $3 d$ printed liver models with computed tomography. Quant Imaging Med Surg. 2019;9:43-52.

4. Sun Z, Lau I, Wong YH, et al. Personalized threedimensional printed models in congenital heart disease. J Clin Med. 2019;8:522.

5. Sun Z, Lee S. A systematic review of 3D printing in cardiovascular and cerebrovascular diseases. Anatol J Cardiol. 2017;17:423-435.

6. Lau I, Sun Z. Dimensional accuracy and clinical value of 3D printed models in congenital heart disease: $A$ systematic review and meta-analysis. J Clin Med. 2019;8:1483.

7. Perica ER, Sun Z. A systematic review of threedimensional printing in liver disease. J Digit Imaging. 2018;31:692-701.

8. Sun Z, Liu D. A systematic review of clinical value of three-dimensional printing in renal disease. Quant Imaging Med Surg. 2018;8:311-325.

9. Perica E, Sun Z. Patient-specific three-dimensional printing for pre-surgical planning in hepatocellular carcinoma treatment. Quant Imaging Med Surg. 2017;7:668-677.

10. Lupulescu C, Sun Z. A systematic review of the clinical value and applications of three-dimensional printing in renal surgery. J Clin Med. 2019;8:990.

11. Loke YH, Harahsheh AS, Krieger A, Olivier L. Usage of 3D models of tetralogy of Fallot for medical education: impact on learning congenital heart disease. BMC Med Educ. 2017;17;54.

12. Su W, Xiao Y, He S, et al. Three-dimensional printing models in congenital heart disease education for medical students: a controlled comparative study. BMC Med Educ. 2018;18(1):178.

13. Ejaz F, Ryan J, Henriksen M, et al. Color-coded patientspecific physical models of congenital heart disease. Rapid Prototyp J. 2014;20:336-343.

14. Wang $\mathrm{Z}$, Liu $\mathrm{Y}$, Luo $\mathrm{H}$, et al. Is a Three-dimensional printing model better than a traditional cardiac model for medical education? A pilot randomized controlled study. Acta Cardiol Sin. 2017;33:664-669.

15. White SC, Sedler J, Jones TW, et al. Utility of threedimensional models in resident education on simple and complex intracardiac congenital heart defects. Congenit Heart Dis. 2018;13(6):1045-1049.

16. Ford S, Minshall T. Invited review article: where and how 3D printing is used in teaching and education. Addit Manuf. 2019;25:131-150.

17. Valverde I, Gomez-Ciriza G, Hussain T, et al. Three dimensional printed models for surgical planning of complex congenital heart defects: an international 
multicenter study. Eur J Cardiothorac Surg. 2017;52:11391148.

18. Anwar S, Rockefeller T, Raptis D, et al. 3D printing provides a precise approach in the treatment of tetralogy of Fallot, pulmonary atresia with major aortopulmonary collateral arteries. Curr Treat Options Cardiovasc Med. 2018;20(1):5.

19. Lau I, Sun Z. Three-dimensional printing in congenital heart disease: A systematic review. J Med Radiat Sc. 2018;65:226-236.

20. Fan $Y$, Wong RHL, Lee AP. Three-dimensional printing in structural heart disease and intervention. Ann Transl Med. 2019;7(20):579.

21. Ripley B, Kelil T, Cheezum MK, et al. 3D printing based on cardiac CT assists anatomic visualization prior to transcatheter aortic valve replacement. J Cardiovasc Comput Tomogr. 2016;10:28-36.

22. Gallo M, D'Onofrio A, Tarantini G, et al. 3D-printing model for complex aortic transcatheter valve treatment. Int J Cardiol. 2016;210:139-40.

23. Lee $\mathrm{AP}$, Leong $\mathrm{CW}$, Kwok $\mathrm{KW}$, et al. Using 3D printed models for planning transcatheter aortic valve implantation in Patients with bicuspid aortic valve. J Am Coll Cardiol. 2018;71:1130.

24. El Sabbagh A, Eleid M, Said S, et al. 3D printing for procedural simulation of transcatheter mitral valve replacement in patients with mitral annular calcification. J Am Coll Cardiol. 2017;69:1142.

25. Fan $Y$, Kwok KW, Zhang Y, et al. Three-Dimensional printing for planning occlusion procedure for a double lobed left atrial appendage. Circ Cardiovasc Interv. 2016;9:e003561.

26. Liu $P$, Liu $R$, Zhang $Y$, et al. The Value of 3D Printing models of left atrial appendage using real-time 3D transesophageal echocardiographic data in left atrial appendage occlusion: applications toward an Era of Truly Personalized Medicine. Cardiology. 2016;135:255-61.

27. Otton JM, Spina R, Sulas $R$, et al. Left atrial appendage closure guided by personalized 3D-printed cardiac reconstruction. JACC Cardiovasc Interv. 2015;8:1004-6.

28. Fan Y, Yang F, Cheng GS, et al. Device sizing guided by echocardiography-based three-dimensional printing is associated with superior outcome after percutaneous left atrial appendage occlusion. J Am Soc Echocardiogr. 2019;32(6):708-719.

29. Wang H, Liu J, Zheng $X$, et al. Three-dimensional virtual surgery models for percutaneous coronary intervention (PCI) optimization strategies. Sci Rep. 2015;5:10945.

30. Lee M, Moharem-Elgamal S, Beckingham R, et al. Evaluating 3D-printed models of coronary anomalies: a survey among clinicians and researchers at a university hospital in the UK. BMJ Open. 2019;9:e025227.
31. Misra A, Walters HL, Kobayashi D. Utilisation of a three-dimensional printed model for the management of coronary-pulmonary artery fistula from left main coronary artery. Cardiol Young. 2019;29:431-434.

32. Oliveira-Santos $M$, Oliveira Santos $E$, Marinho AV, et al. Patient-specific 3D printing simulation to guide complex coronary intervention. Rev Port Cardiol. 2018;37:541.e1-541.e4.

33. Aroney N, Lau K, Danielle L, et al. Three-dimensional printing: to guide management of a right coronary artery to left ventricular fistula. Eur Heart J Cardiovascular Imaging. 2018;19:268.

34. Velasco Forte MN, Byrne N, Valverde Perez I, et al. 3D printed models in patients with coronary artery fistulae: anatomical assessment and interventional planning. Eurolntervention. 2017;13:e1080-e1083.

35. Ho D, Squelch A, Sun Z. Modelling of aortic aneurysm and aortic dissection through 3D printing. J Med Radiat Sc. 2017;64:10-17.

36. Sun Z, Squelch A. Patient-specific 3D printed models of aortic aneurysm and aortic dissection. J Med Imaging Health Inf. 2017;7:886-889.

37. Hossien A, Gesomino S, Maessen J, et al. The interactive use of multi-dimensional modeling and 3D printing in preplanning of type A aortic dissection. J Card Surg. 2016;31:441-445.

38. Huang J, Li G, Wang W, et al. 3D printing guiding stent graft fenestration: A novel technique for fenestration in endovascular aneurysm repair. Vascular. 2017;25:442-446.

39. Bortsman J, Mahmood F, Schermerhorn M, et al. Use of three-dimensional printing to create patientspecific abdominal aortic models for preoperative planning. J Cardiothorac Vasc Anesth. 2019;33(5):1442-1446.

40. Rynio P, Kazimierczak A, Jedrzejczak T, et al. A 3D printed aortic arch template to facilitate decisionmaking regarding the use of an externalized transapical wire during thoracic endovascular aneurysm repair. Ann Vasc Surg. 2019;54:336.e5336.e8.

41. Abdullah KA, McEntee MF, Reed W, Kench PL. Development of an organ-specific insert phantom generated using a 3D printer for investigations of cardiac computed tomography protocols. J Med Radiat Sci. 2018;65:175-183.

42.Sun Z. Personalized three-dimensional printed coronary artery models for accurate assessment of coronary stenosis using high resolution imaging. Australasian Med J. 2019;12:105-109. 
43. Sun Z, Ng CK, Squelch A. Synchrotron radiation computed tomography assessment of calcified plaques and coronary stenosis with different slice thicknesses and beam energies on 3D printed coronary models. Quant Imaging Med Surg. 2019;9:6-22.

44. Sun Z, Jansen S. Personalized 3D printed coronary models in coronary stenting. Quant Imaging Med Surg. 2019;9:1356-1367.

45. Sun Z. 3D printed coronary models offer new opportunities for developing optimal coronary CT angiography protocols in imaging coronary stents. Quant Imaging Med Surg. 2019;9:1350-1355.

46. Aldosari S, Jansen S, Sun Z. Optimization of computed tomography pulmonary angiography protocols using 3D printed model with simulation of pulmonary embolism. Quant Imaging Med Surg. 2019;9:53-62.

47. Aldosari S, Jansen S, Sun Z. Patient-specific 3D printed pulmonary artery model with simulation of peripheral pulmonary embolism for developing optimal computed tomography pulmonary angiography protocols. Quant Imaging Med Surg. 2019;9:75-85.

48.Sun Z. Use of three-dimensional printing in the development of optimal cardiac CT scanning protocols. Curr Med Imaging Rev. 2020. doi: 10.2174/1573405616666200124124140. [Epub ahead of print].

49. Sun Z, Aldosari S. Three-dimensional printing in medicine: Opportunities for development of optimal CT scanning protocols. AMJ. 2018;11(11):529-532.

\section{PEER REVIEW}

Peer reviewed.

\section{CONFLICTS OF INTEREST}

The authors declare that they have no competing interests.

\section{FUNDING}

None

\section{ETHICS COMMITTEE APPROVAL}

Not applicable as this is a review article.
Figure 1: 3D printed coronary models of a patient with congenital heart disease

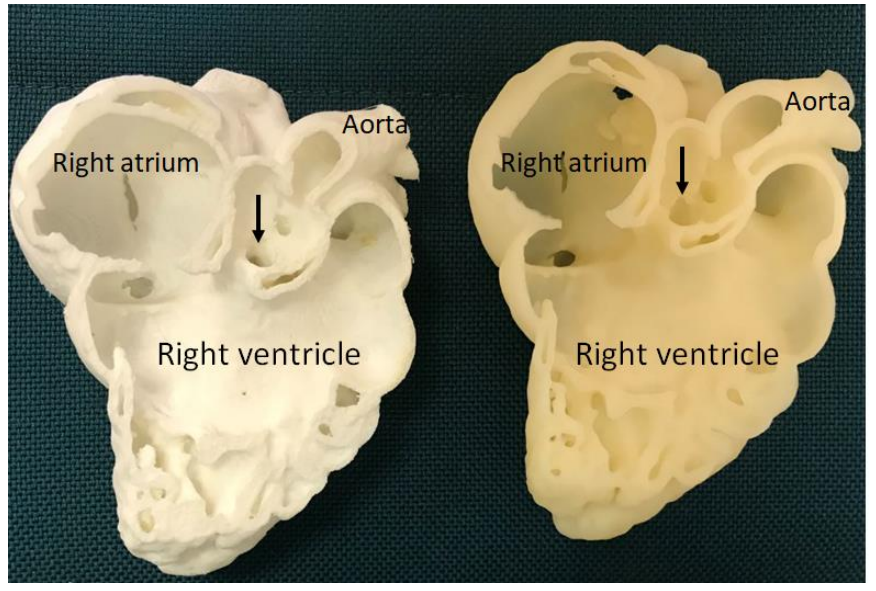

The 3D model was printed with different materials with the left model printing using Thermoplastic polyurethane (TPU) and the right one with Tango Plus. Arrows refer to the ventricular septal defect in this case.

Figure 2: 3D printed model of Stanford type A aortic dissection

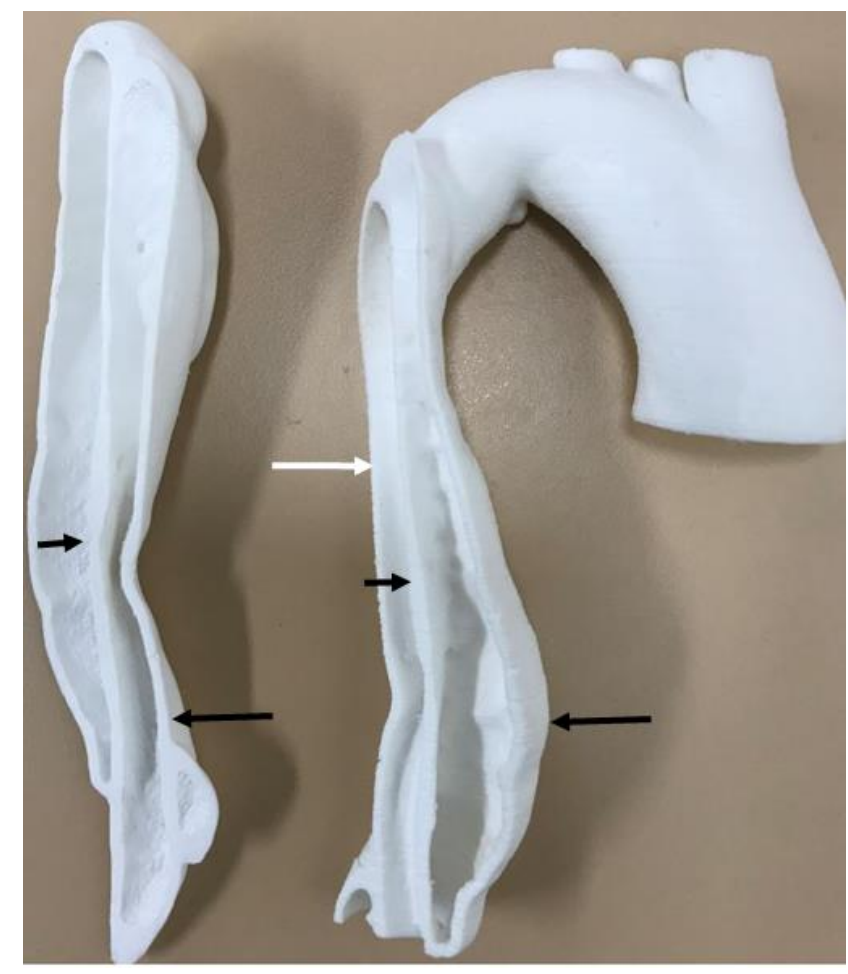

The 3D model was printed to demonstrate the intimal flap (short arrows) which separates the true lumen (white arrow) from the false lumen (black arrows). 
Figure 3: 3D printed model of a normal coronary artery

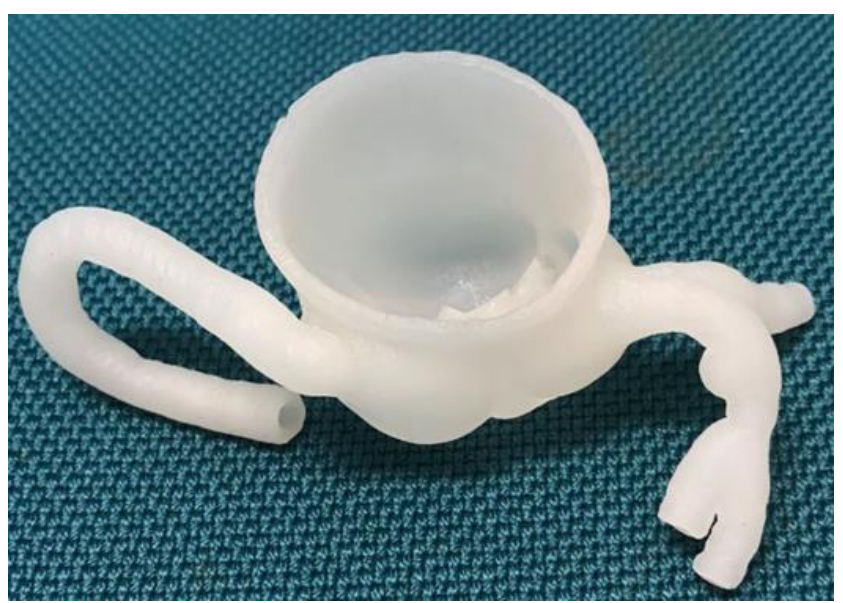

Patient-specific 3D printed coronary model consisting of right and left main coronary arteries.

Figure 4: 3D printed coronary model with simulation of calcified plaques

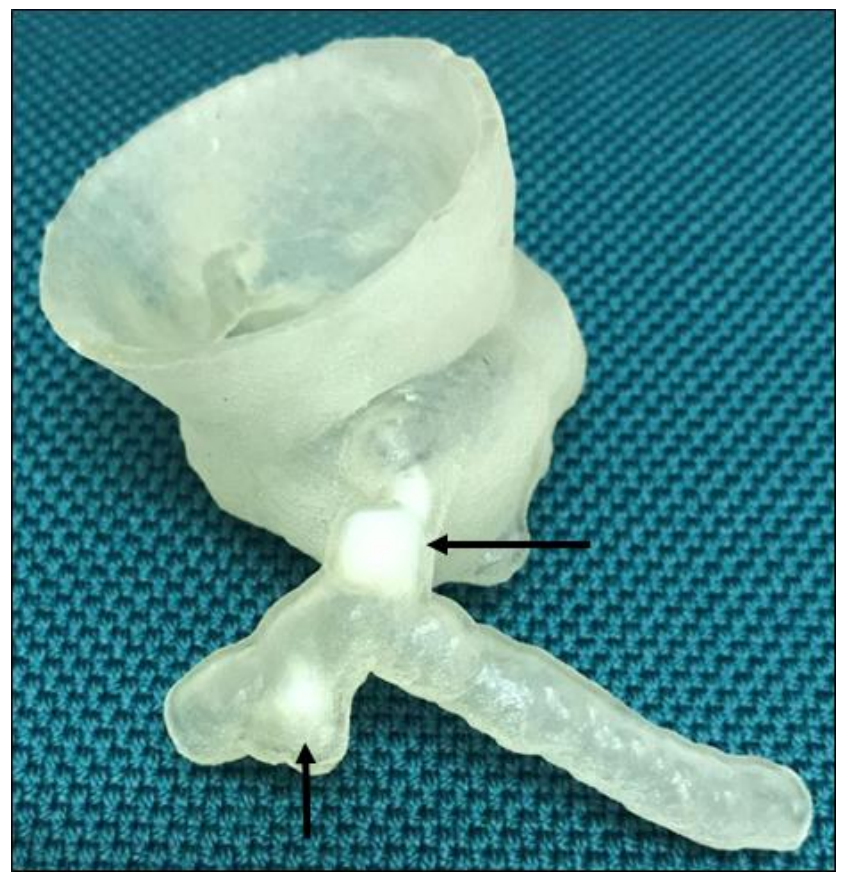

3D printed coronary model printed using soft and elastic Tango Plus material with calcification printed using rigid VeroWhite material. The calcifications were inserted into the left coronary arteries. Long arrow refers to the calcification in the left main stem, while short arrow points to the calcification in the left anterior descending artery. 